\title{
Can Studies of Neuroinflammation in a TSPO Genetic Subgroup (HAB or MAB) Be Applied to the Entire AD Cohort?
}

\author{
Zhen Fan ${ }^{1}$, Denise Harold ${ }^{2}$, Giuseppe Pasqualetti ${ }^{1}$, Julie Williams ${ }^{2}$, David J. Brooks ${ }^{1,3}$, and Paul Edison ${ }^{1,2}$ \\ ${ }^{I}$ Neurology Imaging Unit, Imperial College London, Hammersmith Campus, London, United Kingdom; ${ }^{2}$ MRC Centre for \\ Neuropsychiatric Genetics and Genomics, Cardiff University, Cardiff, United Kingdom; and ${ }^{3}$ Department of Nuclear Medicine, \\ Aarhus University, Denmark
}

Neuroinflammation plays a significant role in Alzheimer disease (AD), and translocator protein (TSPO) PET imaging allows us to quantify this process. However, the binding of second-generation TSPO tracers depends on the TSPO genotype coded by the rs6971 single-nucleotide polymorphism, with a $40 \%-50 \%$ increase in BP in high-affinity binders (HABs) compared with mixed-affinity binders (MABs), whereas low-affinity binders (LABs) are unsuitable for evaluation. Hence, several studies are using either $\mathrm{HAB}$ alone or $\mathrm{HAB}$ and MAB subjects. To translate the findings of neuroinflammation studies to the entire population, it is crucial to establish the influence of TSPO genotypes on AD. Here, we investigated whether different TSPO genotypes influence cognitive function, amyloid load, and disease progression over time. Methods: We evaluated 798 subjects (225 control, 388 with mild cognitive impairment [MCl], and 185 with $A D$ ) from the Alzheimer's Disease Neuroimaging Initiative database at baseline and during follow-up. All subjects were screened for TSPO genotype and underwent detailed clinical and neuropsychologic assessments yearly for $4 \mathrm{y}$. Of the 798 subjects, 255 also had T1- and T2-weighted MR imaging and amyloid PET with ${ }^{11} \mathrm{C}$-Pittsburgh compound $\mathrm{B}$ or ${ }^{18} \mathrm{~F}$-florbetapir. Results: We demonstrated that all TSPO binding groups (HAB, MAB, and LAB) have same level of amyloid load in $A D$ and $\mathrm{MCl}$ subjects. We also demonstrated that the prevalence is $50.3 \%$ for $\mathrm{HAB}, 41.2 \%$ for $M A B$, and $8.5 \%$ for $L A B$, without a statistical difference among the $A D, M C l$, and control groups. During longitudinal follow-up, the mean change in neuropsychometric test scores on the MiniMental State Examination, the cognitive and modified Alzheimer Disease Assessment Scales (ADASs), and the Geriatric Depression Scale over time were similar in $A D$ and $\mathrm{MCl}$ subjects among the 3 TSPO binding groups. Analysis of the covariates showed that diagnostic group (control, $\mathrm{MCl}, \mathrm{AD}$ ), apolipoprotein $\mathrm{E} 4$ status, and sex had a significant effect on decline on the modified Alzheimer Disease Assessment Scale ( $>3$ points of the scale), but age and TSPO genotype did not. Conclusion: This study suggests that information obtained from evaluating a subgroup of $A D$ or $\mathrm{MCl}$ subject using second-generation TSPO tracers can be translated to the entire AD and $\mathrm{MCl}$ population. Thus, we can study fewer $\mathrm{AD}$ subjects in evaluating new antineuroinflammatory and antimicroglial agents in intervention studies and in observational studies evaluating the role of neuroinflammation.

\footnotetext{
Received Oct. 22, 2014; revision accepted Feb. 25, 2015.

For correspondence or reprints contact: Paul Edison, CCTI, Neurology Imaging Unit, Imperial College London, 1st Floor B Block, Hammersmith Hospital Campus, Du Cane Rd., London, W12 ONN, U.K.

E-mail: paul.edison@imperial.ac.uk

Published online Apr. 3, 2015.

COPYRIGHT (c) 2015 by the Society of Nuclear Medicine and Molecular Imaging, Inc.
}

Key Words: Alzheimer's disease; TSPO; MCl; cognitive function; PET study

J Nucl Med 2015; 56:707-713

DOI: 10.2967/jnumed.114.149443

$\mathbf{N}$

euroinflammation plays a significant role in Alzheimer disease $(\mathrm{AD})$ and other neurodegenerative diseases. Studies are evaluating the influence of neuroinflammation on neurodegeneration, and therapeutic agents against these targets are still under clinical evaluation to prevent neurodegeneration in $\mathrm{AD}$ (1). Before commencing a large phase 3 study, it is essential to determine whether these agents can suppress brain inflammation. Neuroinflammation is characterized by an increase in $18-\mathrm{kDa}$ translocator protein (TSPO) expression and is assessed in vivo by the use of TSPO PET. TSPO overexpression after microglial activation (2) can be imaged via binding of PET ligands such as ${ }^{11} \mathrm{C}-(R)-\mathrm{PK} 11195$ (1-[2-chlorophenyl]$N$-methyl- $N$-[1-methyl-propyl]-3-isoquinoline carboxamide). AD subjects and a proportion of subjects with mild cognitive impairment (MCI) show a 20\%-35\% increase in microglial activation in the frontal, temporal, parietal, occipital, and cingulate cortices $(P<$ 0.05) (3-6). Currently, several second-generation TSPO ligands $\left({ }^{11} \mathrm{C}\right.$ PBR28, ${ }^{11} \mathrm{C}-\mathrm{DPA} 713$, and $\left.{ }^{18} \mathrm{~F}-\mathrm{GE} 180\right)(7-9)$ have been developed with the aim of achieving higher affinity and better signal-to-noise ratio than is possible with ${ }^{11} \mathrm{C}-(R)-\mathrm{PK} 11195$ PET. However, their uptake is dependent on TSPO phenotype-high-affinity binders (HABs), low-affinity binders (LABs), and mixed-affinity binders (MABs) $(10,11)$-with a $40 \%-50 \%$ increase in binding potential for HABs compared with MABs, whereas LABs are unsuitable for evaluation. It is now clear that a nonsynonymous polymorphism of TSPO, coded by the rs6971 single-nucleotide polymorphism, affects the binding affinity of these tracers $(10,11)$. This single-nucleotide polymorphism in exon 4 of the TSPO gene causes an alanine-tothreonine substitution in position 147. The Ala/Ala TSPO genotype (wild-type) results in high-affinity binding, whereas Ala/Thr results in mixed-affinity binding, and Thr/Thr results in low-affinity binding. MABs have $40 \%-50 \%$ lower binding potential for TSPO than do HABs (10-12). Because of the lack of binding in LABs, they are unsuitable for evaluation. Hence, several natural progression and intervention studies are evaluating only HAB subjects. Apart from the binding of the PET tracer, TSPO gene mutation was previously found to be associated with a functional alteration that reduces peripheral lymphocyte allopregnanolone production (13). Given 
TABLE 1

Baseline Demography

\begin{tabular}{|c|c|c|c|c|c|c|c|c|c|c|c|}
\hline \multirow[b]{2}{*}{ Group } & \multirow[b]{2}{*}{$n$} & \multirow[b]{2}{*}{ Male (\%) } & \multirow[b]{2}{*}{ Age $^{*}(y)$} & \multicolumn{2}{|c|}{$\begin{array}{l}\text { Amyloid- } \\
\text { positive }\end{array}$} & \multicolumn{6}{|c|}{ Scores } \\
\hline & & & & $\%$ & $n$ & ApoE4 & MMSE $^{\star}$ & ADAS-Cog ${ }^{*}$ & ADAS-Mod* & $\mathrm{CDR}^{\star}$ & $\mathrm{GDS}^{*}$ \\
\hline Control & 225 & 52.0 & $76 \pm 5.0$ & 31 & 99 & 1.8 & $29 \pm 1$ & $6.2 \pm 3$ & $9 \pm 4.1$ & $0.0 \pm 0.0$ & $0.8 \pm 1.1$ \\
\hline $\mathrm{MCl}$ & 388 & 63.9 & $75 \pm 7.3$ & 64 & 137 & 12.4 & $27 \pm 1.8$ & $12 \pm 4.4$ & $18 \pm 6.6$ & $0.49 \pm 0.02$ & $1.6 \pm 1.4$ \\
\hline$A D$ & 185 & 52.9 & $75 \pm 7.5$ & 84 & 19 & 17.9 & $23 \pm 2.0$ & $18 \pm 6.7$ & $28 \pm 9.5$ & $0.74 \pm 0.25$ & $1.6 \pm 1.4$ \\
\hline Total & 798 & 57.4 & $75 \pm 6.8$ & 255 & & 10.6 & $26 \pm 2.7$ & $11 \pm 6.4$ & $18 \pm 9.5$ & $0.41 \pm 0.30$ & $1.3 \pm 1.4$ \\
\hline$P$ & & $<0.01$ & NS & $<0.01$ & & $<0.01$ & $<0.01$ & $<0.01$ & $<0.01$ & $<0.01$ & $<0.01$ \\
\hline $\begin{array}{l}{ }^{*} \text { Data are } \\
\text { ApoE4 = } \\
\text { Amyloid }\end{array}$ & $\begin{array}{l}\text { mean } \\
\text { ApoE } \\
\text { ET sc }\end{array}$ & $\begin{array}{l}\text { SD. } \\
\text { ApoE4 hor } \\
\text { in was perf }\end{array}$ & $\begin{array}{l}\text { ozygous; } \\
\text { rmed on o }\end{array}$ & $\begin{array}{l}S=\text { no } \\
\text { ly } 255\end{array}$ & $\begin{array}{l}\text { tatist } \\
\text { tients }\end{array}$ & $\begin{array}{l}\text { ally sig } \\
\text { and per }\end{array}$ & $\begin{array}{l}\text { icant. } \\
\text { entage rep }\end{array}$ & rted was cal & Jlated for this & ubgroup. & \\
\hline
\end{tabular}

that neurosteroids such as allopregnanolone may play a role in neuronal survival, favoring neurogenesis and preventing apoptosis (14), it is important to establish whether there is a link between the Thr 147 TSPO genotype and AD development and progression, especially if TSPO genotypes become a discriminating factor for selecting AD patients for neuroinflammation studies. To our knowledge, no study has yet evaluated the clinical impact of TSPO genotypes on $\mathrm{AD}$. If the amyloid load and deterioration of cognitive function (clinical progression) were similar in the 3 TSPO subgroups of $\mathrm{AD}$ and MCI subjects, information obtained from HABs alone could then be generalized to all AD or MCI patients. Here, we hypothesized that the distribution of amyloid deposition and the progression of disease would be the same in all TSPO subgroups within the same diagnostic group. We investigated the influence of different TSPO genotypes on amyloid load and neuropsychometric performance at baseline and during follow-up in $\mathrm{AD}, \mathrm{MCI}$, and healthy control subjects.

\section{MATERIALS AND METHODS}

Our study population was drawn from the ADNI database (http:// adni.loni.usc.edu/). The institutional review board approved the study, and all subjects gave written informed consent. A subset of 798 ADNI subjects was selected and was genotyped with TSPO rs6971 as a part of this study. This subset comprised 225 control, $388 \mathrm{MCI}$, and $185 \mathrm{AD}$ subjects. MCI subjects were enrolled if they had Mini-Mental State Examination (MMSE) scores of between 24 and 30 (inclusive), as described in more detail on the ADNI website. Participants underwent apolipoprotein $\mathrm{E}$ (ApoE) genotyping and were monitored longitudinally at 6- to 12-mo intervals for cognitive function with multiple tests, including the MMSE and Alzheimer Disease Assessment Scale (ADAS) (cognitive [ADAS-Cog] and modified [ADAS-Mod]), Clinical Dementia Rating (CDR), and Geriatric Depression Scale (GDS). Among the cohort of 798 subjects, 255 had T1- and T2-weighted MR imaging and PET evaluation for amyloid burden, 188 had ${ }^{18} \mathrm{~F}$-florbetapir PET (15), and 67 had ${ }^{11} \mathrm{C}$-Pittsburgh compound B PET (16).

\section{Image Analysis}

A complete description of MR imaging and amyloid data acquisition is available on the ADNI website (http://www.adni-info.org/Scientists/ ADNIStudyProcedures.aspx). Image analysis was done as described previously with SPM8 (Wellcome Trust Centre for Neuroimaging) using MR imaging for creating the object map (17). For ${ }^{18} \mathrm{~F}$-florbetapir and ${ }^{11} \mathrm{C}$-Pittsburgh compound $\mathrm{B}$, we used 1.36 and 1.4 , respectively, as the thresholds for amyloid positivity for the mean cortical regions of interest normalized to cerebellar standardized uptake value ratio, based on the range of control in previous studies $(18-20)$.

\section{Genetics}

Individuals were genotyped using the 610-quad array (Illumina), as described by Yoder et al. (10). No quality control has been performed on the publicly available ADNI Genome-Wide Association Study dataset; therefore, the data were subjected to quality control filtering before analysis. This filtering retained individuals with missing genotype rates of less than 0.05 , mean autosomal heterozygosity between 0.30 and 0.36 , and mean X-chromosome heterozygosity either less than 0.02 for men or between 0.28 and 0.42 for women.

\section{Statistical Analysis}

Statistical analysis was performed using SPSS Statistics, version 21 (IBM). The effects of demographic variables were evaluated with factorial ANOVA. Associations between groups were evaluated using Pearson correlation analysis. A 2-sample $t$ test for continuous variables and $\chi^{2}$ test for categoric variables were used to compare baseline variables. KaplanMeyer survival analysis was done using an ADAS-Mod increase of more than 3 as the significant cognitive decline event (21). Log-rank MantelCox testing was used to compare survival curves for each genotype. Cox proportional hazards regression was used to identify baseline variables associated with cognitive decline. The sample size of our cohort was sufficient to warrant a power of regression analysis of more than $90 \%$ with an $\alpha$ error of 0.05 .

TABLE 2

Prevalence of the TSPO Genotypes

\begin{tabular}{|c|c|c|c|c|}
\hline \multirow[b]{2}{*}{ Group } & \multicolumn{4}{|c|}{ Genotype } \\
\hline & Thr/Thr & $\mathrm{Ala} / \mathrm{Thr}$ & $\mathrm{Ala} / \mathrm{Ala}$ & Total \\
\hline Control & 21 (9.3\%) & 98 (43.6\%) & $106(47.1 \%)$ & 225 \\
\hline $\mathrm{MCl}$ & $28(7.2 \%)$ & $156(40.2 \%)$ & 204 (52.6\%) & 388 \\
\hline$A D$ & 19 (10.3\%) & 75 (40.5\%) & $91(49.2 \%)$ & 185 \\
\hline Total & $68(8.5 \%)$ & $329(41.2 \%)$ & 401 (50.3\%) & 798 \\
\hline$P^{\star}$ & & & & 0.570 \\
\hline
\end{tabular}

${ }^{\star}$ Pearson $\chi^{2}$ test. 
TABLE 3

Odds Ratio of the TSPO Genotypes, Heterozygote and Homozygote, and Risk of Dementia

\begin{tabular}{lcccc}
\hline \multirow{2}{*}{ Group } & \multicolumn{2}{c}{ Single-nucleotide polymorphism } & & \\
\cline { 2 - 3 } & Thr/Thr, Ala/Thr & Ala/Ala & Odds ratio & $95 \% \mathrm{Cl}$ \\
\hline Control & 119 & 106 & 1.24 (control vs. MCl) & $0.89-1.73$ \\
$\mathrm{MCl}$ & 184 & 204 & 0.87 (MCl vs. AD) & $0.61-1.24$ \\
$\mathrm{AD}$ & 94 & 91 & 1.09 (control vs. AD) & $0.73-1.60$ \\
\hline
\end{tabular}

\section{RESULTS}

Detailed demographic data and baseline parameters are given in Table 1 . Mean age $( \pm \mathrm{SD})$ was $75.3 \pm 6.8 \mathrm{y}$ for the entire cohort and $75.9 \pm 5.0,74.8 \pm 7.3$, and $75.4 \pm 7.5$ for the control, MCI, and $\mathrm{AD}$ groups, respectively. The 3 groups did not significantly differ in age $(P>>0.05)$.

\section{Baseline Data}

The prevalence of genotypes among diagnostic groups is reported in Table 2. The prevalence of these genotypes did not significantly differ among the $\mathrm{AD}, \mathrm{MCI}$, and control groups on $\chi^{2}$ testing $(P=$ 0.691). The prevalence of the Thr allele (both in homozygotes and in heterozygotes) is reported in Table 3 and did not significantly differ among groups $(P>>0.05)$ : the respective odds ratios and $95 \%$ confidence intervals (CIs) were 1.24 and $0.89-1.73$ for the control versus MCI groups, 0.87 and $0.61-1.24$ for the MCI versus $\mathrm{AD}$ groups, and 1.09 and $0.73-1.60$ for the control versus $\mathrm{AD}$ groups.

The mean baseline parameters clustered by TSPO genotype are reported in Table 4. No differences in sex, age, MMSE, ADAS-Cog, ADAS-Mod, or GDS were found among the different TSPO genotypes $(P>>0.05)$ (Fig. 1). The subgroup of 255 subjects who had amyloid scans did not significantly differ from the rest of the cohort in sex and age even when stratified both for diagnostic group and for TSPO genotype. There was no difference in the prevalence of TSPO genotypes in the amyloid subgroup. Within the same diagnostic group, similar distributions of amyloid deposition in $\mathrm{Thr} / \mathrm{Thr}$, $\mathrm{Ala} / \mathrm{Thr}$, and Ala/Ala were found. Examples of individual parametric images of amyloid deposition among diagnostic groups and TSPO genotypes are shown in Figure 2.
As anticipated, a significant difference in the prevalence of subjects with the ApoE4 genotype was found among groups $(P<$ 0.001). Patients with $\mathrm{AD}$ had the greatest percentage of ApoE4 (17.9\%), followed by patients with MCI (12.4\%); $84.2 \%$ of AD patients and $63.5 \%$ of MCI patients were amyloid-positive, whereas $31.3 \%$ of controls were amyloid-positive $(P<0.01)$. TSPO genotypes did not correlate with ApoE4 genotypes. At baseline, as expected, a significant difference between diagnostic groups was found for MMSE, ApoE4, amyloid status, ADAS-Cog, ADASMod, CDR, and GDS $(P<0.01)$ (Table 1$)$.

\section{Longitudinal Data}

The mean decline in MMSE at 36 mo was $-0.08 \pm 0.60,-0.62 \pm$ 2.05 , and $-1.16 \pm 1.88$ for control, MCI, and AD subjects, respectively; the mean annual decline in ADAS-Cog was $0.13 \pm$ $1.23,0.99 \pm 2.68$, and $2.45 \pm 5.63$, respectively; and the mean annual decline in ADAS-Mod was $0.23 \pm 1.8,1.20 \pm 3.1$, and $1.05 \pm$ 8.6, respectively (for each, $P<0.05$ ). No statistical differences $(P>0.05)$ in mean annual decline of MMSE, ADAS-Cog, or ADAS-Mod were found among the different genotypes in the control, MCI, and AD groups, as shown in Figure 3. No statistical differences in mean variation in GDS $(P>0.05)$ were found among the different TSPO genotypes. HAB had a prevalence of $50.3 \%$, MAB $41.2 \%$, and LAB $8.5 \%$, without statistical differences among the $\mathrm{AD}, \mathrm{MCI}$, and control cohorts.

The linear regression analyses of the TSPO genotypes and the mean annual decline in MMSE, ADAS-Cog, and ADAS-Mod are reported in Table 5. The multiregression analysis of mean annual decline in MMSE, ADAS-Cog, and ADAS-Mod are shown in Table 6. The TSPO genotype was not associated with the mean decline in

TABLE 4

Baseline Characteristics of the 3 TSPO Genotypes

\begin{tabular}{|c|c|c|c|c|c|c|c|c|c|c|c|}
\hline \multirow[b]{2}{*}{ Genotype } & \multirow[b]{2}{*}{$n$} & \multirow[b]{2}{*}{ Male (\%) } & \multirow[b]{2}{*}{$\mathrm{Age}^{*}(\mathrm{y})$} & \multicolumn{2}{|c|}{$\begin{array}{l}\text { Amyloid- } \\
\text { positive }\end{array}$} & \multicolumn{6}{|c|}{ Scores } \\
\hline & & & & $\%$ & $n$ & ApoE4 & MMSE* $^{\star}$ & ADAS-Cog* & ADAS-Mod* & $\mathrm{CDR}^{\star}$ & $\mathrm{GDS}^{\star}$ \\
\hline Thr/Thr & 68 & 60.2 & $76.6 \pm 6.1$ & 62.5 & 24 & 16.2 & $26.7 \pm 2.7$ & $11.8 \pm 7.0$ & $18.2 \pm 10.5$ & $0.41 \pm 0.32$ & $1.5 \pm 1.5$ \\
\hline Ala/Thr & 329 & 56.2 & $75.2 \pm 6.7$ & 52.2 & 113 & 8.5 & $26.8 \pm 2.7$ & $11.7 \pm 6.5$ & $18.3 \pm 9.6$ & $0.40 \pm 0.30$ & $1.3 \pm 1.3$ \\
\hline Ala/Ala & 401 & 59.1 & $75.2 \pm 7.0$ & 50.8 & 118 & 11.5 & $26.7 \pm 2.6$ & $11.6 \pm 6.3$ & $18.1 \pm 9.2$ & $0.42 \pm 0.29$ & $1.5 \pm 1.4$ \\
\hline Total & 798 & 58.0 & $75.3 \pm 6.8$ & 52.5 & 255 & 10.7 & $26.7 \pm 2.7$ & $11.6 \pm 6.4$ & $18.1 \pm 9.5$ & $0.41 \pm 0.30$ & $1.4 \pm 1.4$ \\
\hline$P$ & & $>>0.05$ & $>>0.05$ & $>>0.05$ & & $>0.05$ & $>>0.05$ & $>>0.05$ & $>>0.05$ & $>>0.05$ & $>0.05$ \\
\hline
\end{tabular}

${ }^{*}$ Data are mean \pm SD.

ApoE4 = ApoE4/ApoE4 homozygous.

Amyloid PET scan was performed on only 255 patients, and percentage reported was calculated for this subgroup. 


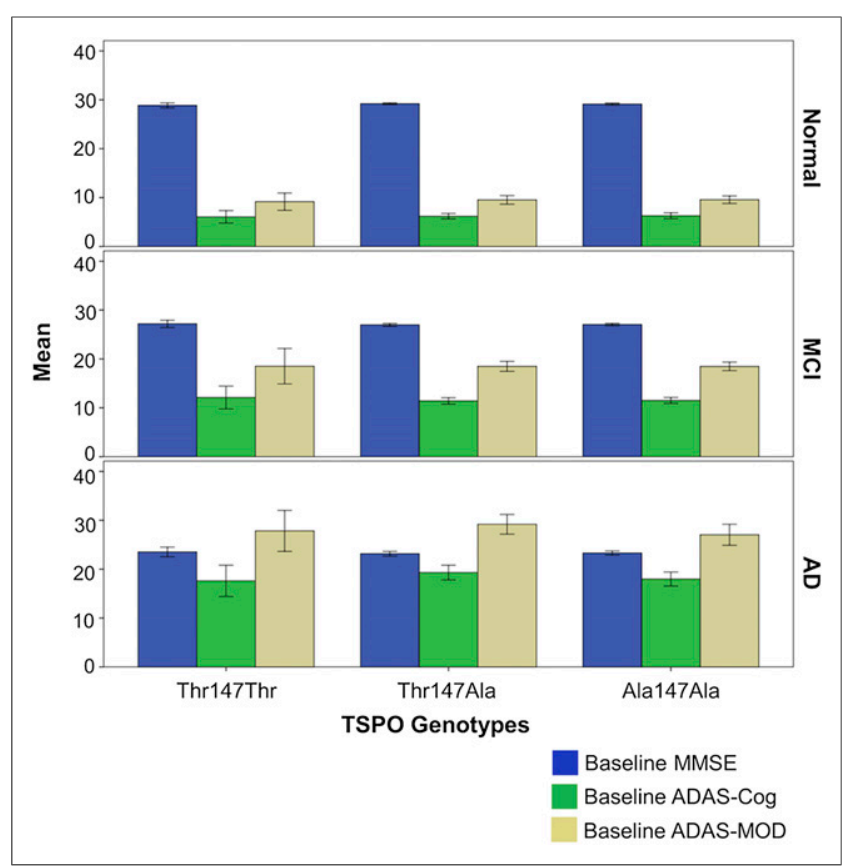

FIGURE 1. Baseline mean MMSE, ADAS-Cog, and ADAS-Mod scores stratified by diagnostic group and genotype. No significant differences were found for any neuropsychologic test across the 3 genotypes $(P>$ 0.05). Error bars show $95 \% \mathrm{Cl}$.

MMSE, ADAS-Cog, or ADAS-Mod. The mean annual decline in ADAS-Mod significantly correlated with age, presence of ApoE4, diagnostic group (control, MCI, and $\mathrm{AD}$ ), and sex. The survival analysis of ADAS-Mod changes ( $>3$ points of the scale) performed on the entire population stratified for genotype did not reveal any significant differences $(P>>0.05)$ on Mantel-Cox testing (Fig. 4). Analysis of the covariates showed a significant effect on ADAS-Mod ( $>3$ points of the scale) for diagnostic group, ApoE4, and sex $(P<$ $0.05)$ but did not show any effect on age or TSPO genotype. MantelCox testing including TSPO genotype as covariate in 134 amyloidpositive subjects did not reveal any significant association (hazard ratio, 1.01 ; 95\% CI, 0.78-1.43; $P=0.72$ ). Repeated analysis adjusting for the presence of ApoE4 did not improve the results for TSPO genotype (ApoE4 allele showed a trend in cognitive decline even if it did not reach statistical significance; hazard ratio, 1.35 ; 95\% CI, $0.90-2.01 ; P=0.16)$.

\section{DISCUSSION}

To our knowledge, this was the first study to evaluate the influence of TSPO genotypes on the clinical phenotypes of $\mathrm{AD}$ and disease progression. We demonstrated that the pathologic features, clinical phenotypes, and rate of cognitive decline in $\mathrm{AD}$ and $\mathrm{MCI}$ are similar in 3 TSPO subgroups. This finding is clinically relevant considering that increasing interest in the role of microglia and neuroinflammation in $\mathrm{AD}$ pathophysiology has led to the development of several novel TSPO radiotracers aimed at clarifying the disease process, improving diagnosis, and marking disease progression $(5,11,22)$. To make the assessments more robust, some studies have chosen to use only subjects who are TSPO HABs, representing around 50\% of Caucasians (12). This approach will result in a selection bias of patients. Moreover, if changes in levels of microglial activation are to be used as a surrogate endpoint in future interventional AD trials,

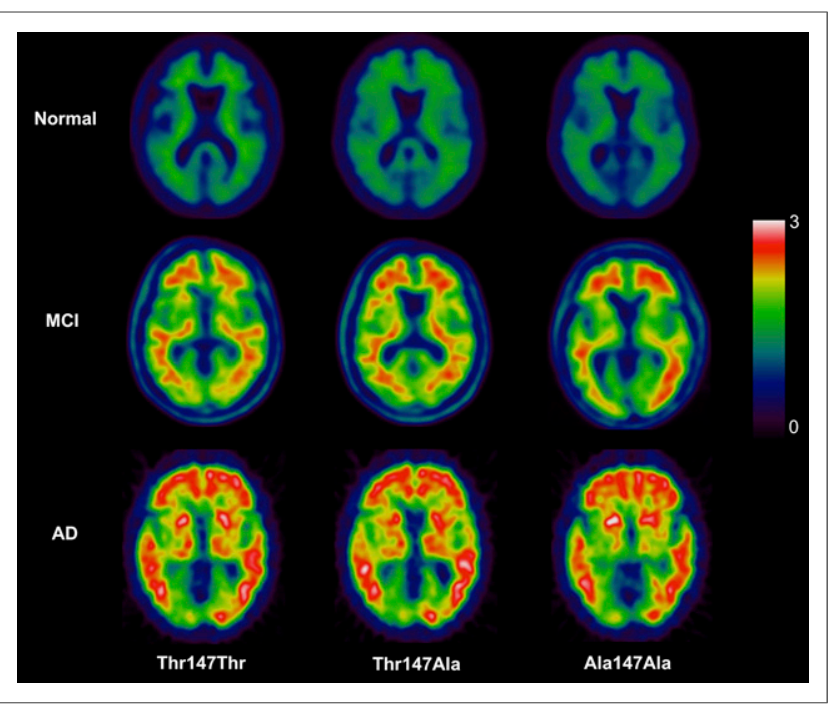

FIGURE 2. Target-to-cerebellar ratio images of amyloid deposition. Each row represents an individual subject from each of the 3 diagnostic groups, and each column represents an individual scan from each of the 3 genotypes.

$50 \%$ of subjects could not be enrolled on the basis of their TSPO genotype. Our data suggest that evaluating a subgroup of AD subjects with HAB will still allow their clinical and amyloid PET results to be translated to the rest of the $\mathrm{AD}$ patients, and considering the sample size in this cohort we can conclude that this finding is robust.

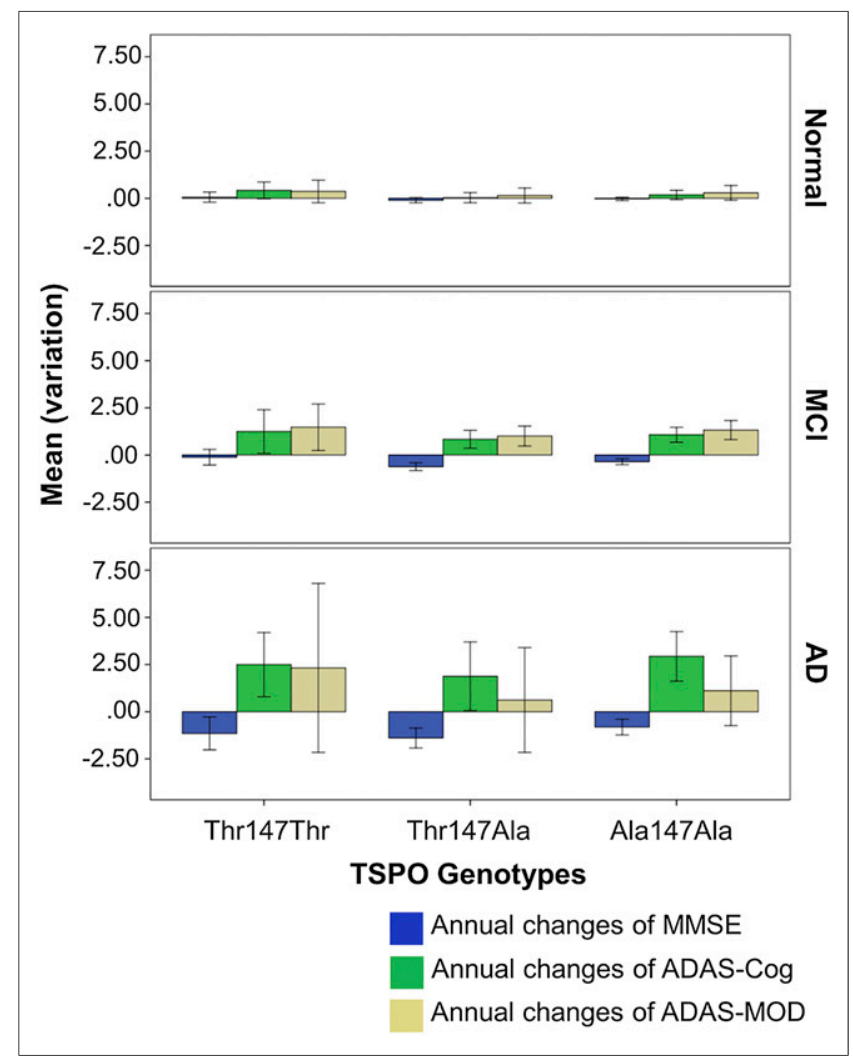

FIGURE 3. Annual decline in mean MMSE, ADAS-Cog, and ADASMod scores stratified by diagnostic group and genotype. No significant differences were found for any neuropsychologic test across the 3 genotypes $(P>0.05)$. Error bars show $95 \% \mathrm{Cl}$. 
TABLE 5

Regression Analysis of TSPO Genotypes and Change in Cognitive Function

\begin{tabular}{|c|c|c|c|c|c|}
\hline Mean annual decline in... & Independent variable (TSPO genotype) & B & SE & $\beta$ & $P$ \\
\hline MMSE & Thr/Thr, Ala/Thr, Ala/Ala & 0.23 & 0.15 & 0.054 & 0.13 \\
\hline ADAS-Cog & Thr/Thr, Ala/Thr, Ala/Ala & -0.16 & 0.16 & -0.035 & 0.32 \\
\hline ADAS-Mod & Thr/Thr, Ala/Thr, Ala/Ala & 0.09 & 0.230 & 0.01 & 0.70 \\
\hline
\end{tabular}

Amyloid burden, although measured with 2 different methods and multiple cameras, was prevalent in the 3 diagnostic groups at a level consistent with the literature (23). Additionally, the prevalence of ApoE4 was the same among the 3 TSPO subgroups in AD, MCI, and control subjects. The prevalence of 3 TSPO phenotypes in AD and MCI was consistent with the prevalence of the TSPO phenotypes in the general population: $49 \%$ for $\mathrm{HAB}, 9 \%$ for $\mathrm{LAB}$, and $42 \%$ for $\operatorname{MAB}(10,12)$.

Longitudinal analysis has been addressed with 2 different methods to improve the quality of the data. We first analyzed the data using linear regression with mean decline over time of the cognitive test scores (MMSE, ADAS-Cog, and ADAS-Mod) as the parameter estimates, and TSPO genotype as the dependent variable. We also performed a multiregression analysis with survival function using several independent variables to assess whether other factors (age, ApoE4, sex, diagnostic group) could better explain cognitive decline. Consistent with baseline analysis, the regression analyses confirmed that the rate of cognitive decline was similar over time (up to $36 \mathrm{mo}$ ) between genotypes and was supported by a similar mean annual decline in change in ADASCog and ADAS-Mod score or MMSE. The multiregression analysis showed similar results within diagnostic groups with different TSPO genotypes, and interestingly, for ADAS-Mod, mean annual decline correlated inversely with age, as previously reported by Holland et al. (24). The Kaplan-Mayer analysis confirmed baseline and linear regression analysis results. The covariate analysis revealed that a significant association existed only between ADAS-Mod increase ( $>3$ points), diagnostic group (MCI, AD, control), ApoE4 status, and sex. There were inconsistencies in the literature regarding the sex differences and the an- nual rate of cognitive decline, but it has been suggested that men (with a larger brain volume) have higher cognitive reserve to withstand more insult (pathologic changes) than women (25), as agrees with our finding. The latter findings have already been published and were not the objective of this study (26-28). Finally, even if underpowered, the subanalysis of TSPO genotypes and the hazards of cognitive worsening in amyloid-positive subjects did not show any significant correlation even after adjusting for the presence of ApoE4 homozygote, thus suggesting that TSPO genotype seems not to have any significant interaction with amyloid deposition.

${ }^{11} \mathrm{C}$-PBR28 has an 80 -fold higher affinity for TSPO than the first-generation TSPO tracer, ${ }^{11} \mathrm{C}-\mathrm{PK} 11195 .{ }^{11} \mathrm{C}-(R)-\mathrm{PK} 11195$ has a significantly lower signal-to-noise ratio than ${ }^{11} \mathrm{C}-\mathrm{PBR} 28$ (29). However, it was found that patients exhibited different binding affinities for TSPO, falling into $\mathrm{HAB}, \mathrm{LAB}$, or MAB groups (29). This variability in binding affects modeling of radioligand binding as a proxy for microglial activation, to the extent that some studies have chosen to use only HABs to make their analysis more robust. Another second-generation ligand is ${ }^{11} \mathrm{C}$-DAA1106, which has much higher affinity for TSPO than ${ }^{11} \mathrm{C}-(R)$-PK11195 and is also influenced by TSPO polymorphisms (30). As more TSPO tracers are being used to evaluate neuroinflammation, our results demonstrating that TSPO genotype did not influence the amyloid pathology or disease progression in $\mathrm{AD}$ is relevant to evaluating AD patients with second-generation TSPO tracers.

At a molecular level, the TSPO protein is involved in steroidogenic cells controlling the uptake of cholesterol and thus the synthesis of neuroactive steroids (31). Allopregnanolone, an endogenous neurosteroid synthesized in the central and peripheral

TABLE 6

Multiregression Analysis of Model Parameter as Independent Variable

\begin{tabular}{|c|c|c|c|c|c|c|c|c|c|c|c|c|}
\hline \multirow[b]{2}{*}{ Model } & \multicolumn{5}{|c|}{ Mean annual MMSE decline } & \multicolumn{4}{|c|}{$\begin{array}{c}\text { Mean annual ADAS-Cog } \\
\text { decline }\end{array}$} & \multicolumn{3}{|c|}{$\begin{array}{l}\text { Mean annual } \\
\text { ADAS-Mod } \\
\text { decline }\end{array}$} \\
\hline & $\mathrm{B}$ & SE & $\beta$ & $P$ & $\mathrm{~B}$ & SE & $\beta$ & $P$ & B & SE & $\beta$ & $P$ \\
\hline Age & 0.01 & 0.01 & 0.04 & 0.30 & 0.01 & 0.01 & 0.02 & 0.62 & 0.04 & 0.02 & 0.07 & 0.05 \\
\hline ApoE4 & -0.34 & 0.20 & -0.06 & 0.09 & 0.22 & 0.21 & 0.04 & 0.30 & 0.69 & 0.30 & 0.09 & 0.02 \\
\hline Diagnostic group (control, $\mathrm{MCl}, \mathrm{AD}$ ) & -1.16 & 0.14 & -0.30 & 0.00 & 1.52 & 0.14 & 0.37 & 0.00 & 1.16 & 0.21 & 0.20 & 0.00 \\
\hline Sex & -0.09 & 0.20 & -0.02 & 0.65 & 0.61 & 0.20 & 0.10 & 0.00 & 0.64 & 0.29 & 0.08 & 0.03 \\
\hline TSOP genotype (Thr/Thr, Ala/Thr, Ala/Ala) & 0.26 & 0.15 & 0.06 & 0.08 & -0.18 & 0.15 & -0.04 & 0.24 & 0.07 & 0.22 & 0.01 & 0.75 \\
\hline
\end{tabular}




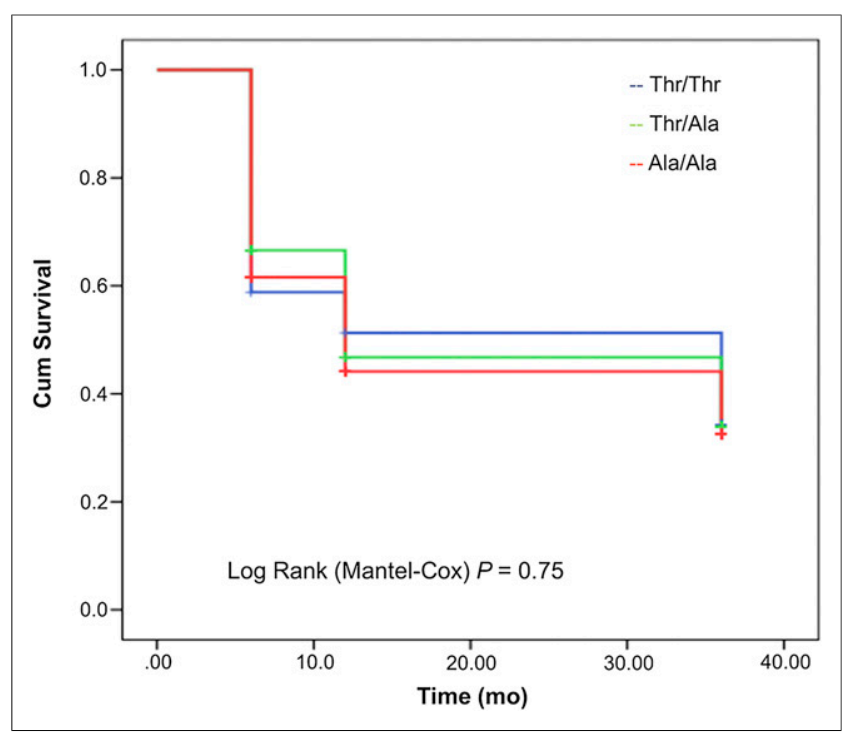

FIGURE 4. Kaplan-Mayer curves of survival analysis for the 3 diagnostic groups when ADAS-Mod increased by more than 3.0 points over time. Data are stratified by genotype. Cum $=$ cumulative.

nervous systems in myelinating glial cells, astrocytes, and several neuronal cell types, declines with age and neurodegenerative disease (14). Costa et al. demonstrated that the Ala/Thr 147 spontaneous amino acid substitution within TSPO is able to affect pregnenolone production in a peripheral cell model, represented by circulating lymphomonocytes (13). However, it is not known to what extent TSPO genotype could affect neurosteroid synthesis such as allopregnanolone at a central nervous system level or whether it could play a role in neurodegenerative pathophysiology. Considering the number of subjects analyzed in our study at baseline and during follow-up, it seems unlikely that TSPO genotype could affect the course of the disease in AD.

A possible limitation of our study is the short follow-up. However, the length of follow-up was reasonable given the short life expectancy of $\mathrm{AD}$ patients (mean, $10 \mathrm{y}$ ) and the annual rate of conversion of $\mathrm{MCI}$ to $\mathrm{AD}(10 \%-15 \%)$. One could argue that, for novel therapeutic targets, the influence of genotype is still unknown. However, unless new potential treatments interfere with TSPO genotypes and cholesterol metabolism, it is unlikely that genotype will have an effect. Even though we have not evaluated the influence of $\mathrm{HAB}, \mathrm{MAB}$, and LAB in other neurodegenerative diseases, it is unlikely that this difference in binding is going to be clinically significant in other conditions.

\section{CONCLUSION}

We have demonstrated that all binding groups (HAB, MAB, and $\mathrm{LAB}$ ) have the same level of amyloid deposition in AD and MCI subjects. The rate of cognitive decline was also similar in the 3 TSPO subgroups of AD and MCI subjects. We have demonstrated that the prevalence of $\mathrm{HAB}$ was $50.3 \%$, whereas $\mathrm{MAB}$ was $41.2 \%$ and $\mathrm{LAB}$ was $8.5 \%$, in $\mathrm{AD}, \mathrm{MCI}$, and control subjects. This finding implies that any information about the $\mathrm{AD}$ process or effects of intervention explored in a subgroup of $\mathrm{HAB}$ or MAB subjects using second-generation TSPO could be translated to the whole AD or MCI population. Thus, fewer AD subjects would need to be studied in evaluations of new antineuroinflammatory and antimicroglial agents in intervention studies and in observational studies evaluating the role of neuroinflammation.

\section{DISCLOSURE}

The costs of publication of this article were defrayed in part by the payment of page charges. Therefore, and solely to indicate this fact, this article is hereby marked "advertisement" in accordance with 18 USC section 1734. This research was supported by Alzheimer's Research U.K. and, in part, by funding from the University of Pisa and from PAIM (Pisa, Italy); research travel costs from the Italian Society of Pharmacology; and grant funding from the Medical Research Council, U.K., Alzheimer's Research, U.K., the Alzheimer's Society, U.K., Novo Nordisk, and GE Healthcare. David J. Brooks was the chief medical officer for GE Healthcare and has received consultancy fees or honoraria from the following: Acadia Pharmaceuticals Inc., Amsterdam Molecular Therapeutics BV, AstraZeneca, Biogen Idec, NeuroNova AB, Eli Lilly and Company, Medtronic Inc., Shire Pharmaceuticals Inc., Synosia Therapeutics AG, GlaxoSmithKline, UBC Biosciences Inc., Veralis (R\&D) Limited, Genentech Inc., and Navidea. No other potential conflict of interest relevant to this article was reported.

\section{ACKNOWLEDGEMENT}

The data used in the preparation of this article were obtained from the Alzheimer's Disease Neuroimaging Initiative database (adni.loni.ucla.edu).

\section{REFERENCES}

1. Lyman M, Lloyd DG, Ji X, Vizcaychipi MP, Ma D. Neuroinflammation: the role and consequences. Neurosci Res. 2014;79:1-12.

2. Nothdurfter C, Baghai TC, Schule C, Rupprecht R. Translocator protein (18 $\mathrm{kDa}$ (TSPO) as a therapeutic target for anxiety and neurologic disorders. Eur Arch Psychiatry Clin Neurosci. 2012;262(suppl 2):S107-S112.

3. Edison P, Archer HA, Hinz R, et al. Amyloid, hypometabolism, and cognition in Alzheimer disease: an $\left[{ }^{11} \mathrm{C}\right] \mathrm{PIB}$ and $\left[{ }^{18} \mathrm{~F}\right] \mathrm{FDG}$ PET study. Neurology. 2007;68: 501-508.

4. Schuitemaker A, Kropholler MA, Boellaard R, et al. Microglial activation in Alzheimer's disease: an (R)-[ $\left.{ }^{11} \mathrm{C}\right] \mathrm{PK} 11195$ positron emission tomography study. Neurobiol Aging. 2013;34:128-136.

5. Edison P, Archer HA, Gerhard A, et al. Microglia, amyloid, and cognition in Alzheimer's disease: an $\left[{ }^{11} \mathrm{C}\right](\mathrm{R}) \mathrm{PK} 11195$-PET and $\left[{ }^{11} \mathrm{C}\right] \mathrm{PIB}-\mathrm{PET}$ study. Neurobiol Dis. 2008;32:412-419.

6. Okello A, Koivunen J, Edison P, et al. Conversion of amyloid positive and negative MCI to AD over 3 years: an ${ }^{11}$ C-PIB PET study. Neurology. 2009;73: 754-760.

7. Guo Q, Owen DR, Rabiner EA, Turkheimer FE, Gunn RN. Identifying improved TSPO PET imaging probes through biomathematics: the impact of multiple TSPO binding sites in vivo. Neuroimage. 2012;60:902-910.

8. Venneti S, Lopresti BJ, Wang G, et al. A comparison of the high-affinity peripheral benzodiazepine receptor ligands DAA1106 and (R)-PK11195 in rat models of neuroinflammation: implications for PET imaging of microglial activation. J Neurochem. 2007;102:2118-2131.

9. Wadsworth H, Jones PA, Chau WF, et al. $\left[{ }^{18} \mathrm{~F}\right] \mathrm{GE}-180$ : a novel fluorine-18 labelled PET tracer for imaging translocator protein $18 \mathrm{kDa}$ (TSPO). Bioorg Med Chem Lett. 2012;22:1308-1313.

10. Yoder KK, Nho K, Risacher SL, Kim S, Shen L, Saykin AJ. Influence of TSPO genotype on ${ }^{11} \mathrm{C}-\mathrm{PBR} 28$ standardized uptake values. J Nucl Med. 2013;54:13201322.

11. Owen DR, Yeo AJ, Gunn RN, et al. An 18-kDa translocator protein (TSPO) polymorphism explains differences in binding affinity of the PET radioligand PBR28. J Cereb Blood Flow Metab. 2012;32:1-5.

12. Kreisl WC, Jenko KJ, Hines CS, et al. A genetic polymorphism for translocator protein $18 \mathrm{kDa}$ affects both in vitro and in vivo radioligand binding in human brain to this putative biomarker of neuroinflammation. J Cereb Blood Flow Metab. 2013;33:53-58.

13. Costa B, Pini S, Gabelloni P, et al. The spontaneous Ala147Thr amino acid substitution within the translocator protein influences pregnenolone production in lymphomonocytes of healthy individuals. Endocrinology. 2009;150:5438-5445. 
14. Irwin RW, Brinton RD. Allopregnanolone as regenerative therapeutic for Alzheimer's disease: translational development and clinical promise. Prog $\mathrm{Neu}$ robiol. 2014;113:40-55.

15. Villemagne VL, Mulligan RS, Pejoska S, et al. Comparison of ${ }^{11} \mathrm{C}-\mathrm{PiB}$ and ${ }^{18} \mathrm{~F}-$ florbetaben for Abeta imaging in ageing and Alzheimer's disease. Eur J Nucl Med Mol Imaging. 2012;39:983-989.

16. Villemagne VL, Pike K, Pejoska S, et al. ${ }^{11} \mathrm{C}-\mathrm{PiB}$ PET ABri imaging in WorsterDrought syndrome (familial British dementia): a case report. J Alzheimers Dis. 2010;19:423-428.

17. Edison P, Carter SF, Rinne JO, et al. Comparison of MRI based and PET template based approaches in the quantitative analysis of amyloid imaging with PIBPET. Neuroimage. 2013;70:423-433.

18. Teipel S, Heinsen H, Amaro E Jr, et al. Cholinergic basal forebrain atrophy predicts amyloid burden in Alzheimer's disease. Neurobiol Aging. 2014;35: $482-491$.

19. Devanand DP, Mikhno A, Pelton GH, et al. Pittsburgh compound B ( $\left.{ }^{11} \mathrm{C}-\mathrm{PIB}\right)$ and fluorodeoxyglucose $\left({ }^{18} \mathrm{~F}-\mathrm{FDG}\right) \mathrm{PET}$ in patients with Alzheimer disease, mild cognitive impairment, and healthy controls. J Geriatr Psychiatry Neurol. 2010;23: 185-198.

20. Landau SM, Lu M, Joshi AD, et al. Comparing positron emission tomography imaging and cerebrospinal fluid measurements of beta-amyloid. Ann Neurol. 2013;74:826-836.

21. Schrag A, Schott JM. Alzheimer's Disease Neuroimaging I: what is the clinically relevant change on the ADAS-Cog? J Neurol Neurosurg Psychiatry. 2012;83: 171-173.

22. Fiala M, Veerhuis R. Biomarkers of inflammation and amyloid-beta phagocytosis in patients at risk of Alzheimer disease. Exp Gerontol. 2010;45:57-63.
23. Quigley H, Colloby SJ, O'Brien JT. PET imaging of brain amyloid in dementia: a review. Int J Geriatr Psychiatry. 2011;26:991-999.

24. Holland D, Desikan RS, Dale AM, McEvoy LK. Alzheimer's Disease Neuroimaging I: rates of decline in Alzheimer disease decrease with age. PLOS ONE. 2012;7:e42325.

25. Mielke MM, Vemuri P, Rocca WA. Clinical epidemiology of Alzheimer's disease: assessing sex and gender differences. Clinical Epidemiology. 2014;6:37-48.

26. Tschanz JT, Corcoran CD, Schwartz S, et al. Progression of cognitive, functional, and neuropsychiatric symptom domains in a population cohort with Alzheimer dementia: the Cache County Dementia Progression Study. Am J Geriatr Psychiatry. 2011;19:532-542.

27. Ward A, Crean S, Mercaldi CJ, et al. Prevalence of apolipoprotein E4 genotype and homozygotes (APOE e4/4) among patients diagnosed with Alzheimer's disease: a systematic review and meta-analysis. Neuroepidemiology. 2012; 38:1-17.

28. Paradise M, Cooper C, Livingston G. Systematic review of the effect of education on survival in Alzheimer's disease. Int Psychogeriatr. 2009;21:25-32.

29. Kreisl WC, Fujita M, Fujimura Y, et al. Comparison of $\left[{ }^{11} \mathrm{C}\right]-(\mathrm{R})-\mathrm{PK} 11195$ and $\left[{ }^{11} \mathrm{C}\right] \mathrm{PBR} 28$, two radioligands for translocator protein $(18 \mathrm{kDa})$ in human and monkey: implications for positron emission tomographic imaging of this inflammation biomarker. Neuroimage. 2010;49:2924-2932.

30. Maeda J, Suhara T, Zhang MR, et al. Novel peripheral benzodiazepine receptor ligand $\left[{ }^{11} \mathrm{C}\right] \mathrm{DAA} 1106$ for PET: an imaging tool for glial cells in the brain. Synapse. 2004;52:283-291.

31. Rupprecht R, Papadopoulos V, Rammes G, et al. Translocator protein (18 kDa) (TSPO) as a therapeutic target for neurological and psychiatric disorders. Nat Rev Drug Discov. 2010;9:971-988. 